\title{
ÍNDICES DE DESEMPENHO ZOOTÉCNICO E ECONÔMICO DE SISTEMAS DE PRODUÇÃO DE LEITE COM DIFERENTES TIPOS DE MÃO DE OBRA
}

\section{ZOOTECHNICAL AND ECONOMIC PERFORMANCE INDEX OF MILK PRODUCTION SYSTEMS WITH DIFFERENT TYPES OF LABOR}

\author{
Rodrigo de Andrade Ferrazza ${ }^{1}$ \\ Marcos Aurélio Lopes ${ }^{2}$ \\ Fábio Raphael Pascoti Bruhn ${ }^{1}$ \\ Flávio de Moraes ${ }^{1}$ \\ ${ }^{1}$ Pós-graduandos da Universidade Federal de Lavras, Lavras, MG, Brasil. \\ 2Professor Doutor da Universidade Federal de Lavras, Lavras, MG, Brasil - malopes@dmv.ufla.br
}

\begin{abstract}
Resumo
Objetivou-se com este estudo estimar índices de desempenho zootécnico e econômico de referência, analisar a influência dos tipos de mão de obra sobre a rentabilidade da atividade e identificar os componentes que exerceram maiores representatividades sobre o custo total e operacional efetivo. Foram analisados 38 índices de tamanho, zootécnico e econômico de 61 sistemas de produção de leite, localizados em Minas Gerais e Rio de Janeiro, agrupados em função do tipo de mão de obra. Os dados foram coletados durante o período de janeiro de 2002 a dezembro de 2011. Os resultados foram comparados pelo teste de ANOVA (distribuição normal), complementado pelo teste LSD, e pelo teste de Kruskal-Wallis (distribuição não normal), também complementado pelo teste LSD. Índices de medição de desempenho de referências devem ser considerados, levando-se em conta os fatores que sejam comuns aos sistemas de produção. O tipo de mão de obra não parece determinar a eficiência econômica do sistema de produção. Os itens componentes do custo total e operacional efetivo que exerceram maiores representatividades foram, em ordem decrescente, alimentação e mão de obra, para os grupos mão de obra contratada e mista, e alimentação e despesas diversas, para o grupo mão de obra familiar.
\end{abstract}

Palavras-chave: análise de rentabilidade; eficiência técnica; índices de referência; pecuária leiteira; recursos humanos.

\begin{abstract}
The objective of this research was to estimate reference zootechnical and economic performance indices, evaluate the influence of different types of labor on the technical and economic results and identify the components that had greater representativeness on the total and effective operational cost of dairy farms. We analyzed 38 size, zootechnical, and economic indicators of 61 milk production systems, grouped according to the type of labor. The data were collected during January 2002 to December 2011 period. The results were compared by ANOVA test (normal distribution), complemented by LSD test, and Kruskal-Wallis test (nonnormal distribution), also complemented by LSD test. Reference performance indices should be considered, taking into account the factors that are common to dairy farms. The type of labor does not seem to determine
\end{abstract}


the economic efficiency of the dairy production system. The items of total and effective operational cost that had greater representativeness were, in descending order, feeding and labor to hired and mixed labor groups, and feeding and various expenses to family labor.

Keywords: benchmarks; dairy cattle; human resources; profitability analysis; technical efficiency.

Recebido em: 14 agosto 2013.

Aceito em: 01 dezembro 2014.

\section{Introdução}

A atividade leiteira é praticada em todas as regiões do Brasil e está presente em aproximadamente 1,8 milhões de propriedades rurais, das quais $80 \%$ são unidades familiares de produção. $\mathrm{O}$ segmento é considerado de grande potencial para a ocupação de mão de obra, pois, para cada U\$2.500,00 vendidos de leite e derivados, é gerado um posto de trabalho permanente ${ }^{(1)}$. Estima-se que o setor envolva cerca de 3,6 milhões de pessoas, produzindo aproximadamente 25 bilhões de litros de leite por ano, provenientes de um dos maiores rebanhos do mundo. No período de 1995 2008, o produto lácteo nacional registrou um aumento de $74,7 \%$ no volume de leite produzido (mil litros) e de $407,5 \%$ no valor da produção ${ }^{(2)}$.

A mão de obra tem uma representatividade significativa na estrutura do custo de produção da maioria dos empreendimentos pecuários do país, com importância crescente em função do aumento dos gastos com a mão de obra, refletido pela evolução histórica do valor real do salário mínimo ${ }^{(3)} \mathrm{e}$ da escassez de trabalhadores qualificados no campo.

Alves et al. ${ }^{(4)}$, ao analisarem 963 estabelecimentos em todo território nacional, na tentativa de explicar a causa de $74 \%$ dos mesmos terem apresentado renda líquida negativa, com base nos insumos terra, capital e trabalho, concluíram que a má alocação de recursos contribuiu de forma significativa para determinar a existência de renda líquida negativa na maior parte da amostra. Nesse mesmo estudo, verificou-se que, em relação ao fator trabalho, 67,5\% usavam em excesso esse fator, ao passo que 19,8\% podiam aumentar os níveis de mão de obra para auferir maior renda e, ainda, que apenas $12,7 \%$ utilizaram o nível ótimo do fator trabalho.

Gonçalves et al. ${ }^{(5)}$ analisaram a eficiência técnica de produtores de leite do estado de Minas Gerais e correlacionaram positivamente a produtividade da mão de obra com a eficiência técnica da atividade leiteira, independentemente do nível de produção. Para esses autores, o impacto da produtividade da mão de obra na eficiência técnica foi maior para os pequenos produtores $(<50$ litros/dia), o que reflete a importância desse quesito para esse grupo de produtores.

Levando em consideração a grande heterogeneidade da cadeia produtiva do leite no Brasil, sua presença em todo o território nacional e o caráter dinâmico inerente ao ambiente de produção ${ }^{(6)}$, a identificação, a quantificação e o estabelecimento de índices técnico-gerenciais e econômicos de referência são de interesse prático para o meio profissional, devendo ser considerados por gestores de empresas rurais e profissionais que trabalham com assistência técnica e extensão rural. A comparação entre os índices alcançados pelo produtor e os obtidos em diferentes sistemas de produção, considerando os fatores que sejam comuns entre os sistemas, pode possibilitar o embasamento para a elaboração de um planejamento, o estabelecimento de metas, a avaliação dos resultados e a tomada de decisões estratégicas.

Diversos critérios de classificação dos sistemas de produção de leite podem ser adotados, e sua 
escolha depende dos objetivos propostos. Dentre os fatores que podem influenciar a viabilidade da atividade está o tipo de mão de obra adotado pelos pecuaristas. Objetivou-se, com esta pesquisa, estimar índices de desempenho zootécnico e econômico de referência, visando auxiliar técnicos e pecuaristas no suporte à tomada de decisões gerenciais em fazendas produtoras de leite. Pretendeuse, ainda, analisar a influência do tipo de mão de obra na rentabilidade da atividade leiteira e identificar os componentes que exerceram maiores representatividades sobre o custo total e operacional efetivo.

\section{Material e métodos}

Foram estimados e analisados os índices de tamanho, zootécnico e econômico de 61 sistemas de produção de leite localizados nas regiões central, sul e sudoeste de Minas Gerais e noroeste, norte e sul do Rio de Janeiro. A amostragem não probabilística por julgamento foi realizada considerandose a disponibilidade e a qualidade dos dados por parte dos produtores.

Para atender aos objetivos da pesquisa e obter melhor interpretação dos resultados, os sistemas de produção foram alocados em função do tipo de mão de obra, denominado nesta pesquisa como familiar (exclusivamente familiar, com contração de mão de obra esporádica para trabalhos eventuais), mista (participação de mão de obra familiar e contratada durante todo o período) e contratada (mão de obra exclusivamente contratada) ${ }^{(7)}$. A expressão mão de obra familiar foi empregada para designar um tipo de sistema de produção em que o trabalho referente às atividades com os animais (ordenha, alimentação, manejo, administração etc.) foi realizado pelo produtor e/ou sua família. Cabe ressaltar que foram imputados valores à remuneração da mão de obra familiar, correspondentes ao salário mínimo vigente na época, visando-se contabilizar os custos de oportunidade da mão de obra pois, embora não haja a efetuação do gasto, o produtor e os familiares estão abrindo mão de outras atividades para dedicarem-se à produção de leite, o que gera custos implícitos.

Os itens que compuseram o custo operacional efetivo de produção do leite foram divididos em grupos: alimentação, mão de obra, sanidade, reprodução, ordenha, impostos considerados fixos, energia e despesas diversas ${ }^{(8)}$. No caso das propriedades que utilizavam hormônio BST, as despesas referentes a esse item foram alocadas no grupo sanidade, enquanto aluguel de máquinas, em despesas diversas.

Os seguintes índices foram avaliados: 1) produção diária de leite (L/dia); 2) área ocupada com a atividade leiteira (ha); 3) mão de obra contratada (dh - dia homem); 4) mão de obra familiar (dh); 5) quantidade de vacas em lactação (cab - cabeças); 6) taxa de lotação de vacas em lactação (cab/ha) = quantidade de vacas em lactação/área total; 7) produtividade por vaca em lactação (L/ano) = produção diária de leite/quantidade de vacas em lactação; 8) vacas em lactação por dia/homem $(\mathrm{cab} / \mathrm{dh})=$ quantidade de vacas em lactação/total de mão de obra anual; 9) produtividade da mão de obra $(\mathrm{L} / \mathrm{dh})=$ produção anual de leite/total de mão de obra anual; 10) produtividade da terra $(\mathrm{L} / \mathrm{ha} / \mathrm{ano})=$ produção anual de leite/área ocupada com a atividade leiteira; 11$)$ receita bruta do leite $(\mathrm{RBL})$ em relação à receita bruta total $(\mathrm{RBT})(\%)=\mathrm{RBL} / \mathrm{RBT} \times 100 ; 12)$ representatividade do custo operacional efetivo $(\mathrm{COE})$ na receita bruta total $(\%)=(\mathrm{COE} / \mathrm{RBT}) \mathrm{x} 100 ; 13)$ representatividade do custo operacional total $(\mathrm{COT})$ na receita bruta total $(\%)=(\mathrm{COT} / \mathrm{RBT}) \times 100$; 14) COE unitário em relação ao preço de leite $(\mathrm{PL})(\%)=(\mathrm{COEun} / \mathrm{PL}) \times 100 ; 15)$ COT unitário em 
relação ao preço de leite $(\%)=($ COTun/PL $)$ x $100 ; 16)$ custo total unitário em relação ao preço de leite $(\%)=(\mathrm{CTun} / \mathrm{PL}) \times 100 ; 17)$ relação entre o custo fixo e o custo total $(\%)=(\mathrm{CF} / \mathrm{CT}) \times 100$ $(\%)$; 18) representatividade da depreciação no COT $(\%)=($ depreciação/COT $)$ x $100 ; 19)$ lucratividade $1(\%)=$ (resultado/receita total) $\mathrm{x} 100 ; 20$ ) lucratividade $2(\%)=$ (margem líquida/receita total) x $100 ; 21)$ rentabilidade $1(\%)=($ resultado/total imobilizado) $\times 100 ; 22)$ rentabilidade $2(\%)=$ (margem líquida/total imobilizado) $x$ 100; 23) representatividade da alimentação no custo total $(\%)=($ alimentação/CT $)$ x $100 ; 24)$ representatividade da mão de obra no custo total $(\%)=($ mão de obra/CT $) \times 100 ; 25)$ representatividade da sanidade no custo total $(\%)=$ $($ sanidade/CT $) \times 100 ; 26)$ representatividade da ordenha no custo total $(\%)=($ ordenha/CT $) \times 100$; 27) representatividade da reprodução no custo total $(\%)=($ reprodução/CT) $x$ 100; 28) representatividade da energia no custo total $(\%)=($ energia/CT $) \times 100 ; 29)$ representatividade dos impostos e taxas no custo total $(\%)=($ impostos e taxas/CT) $x 100 ; 30)$ representatividade das despesas diversas no custo total $(\%)=($ despesas diversas/CT $) \times 100 ; 31)$ representatividade da alimentação no $\operatorname{COE}(\%)=($ alimentação/COE) x $100 ; 32)$ representatividade da mão de obra no $\operatorname{COE}(\%)=($ mão de obra/COE $) \times 100 ; 33)$ representatividade da sanidade no $\mathrm{COE}(\%)=$ $($ sanidade/COE $) \times 100 ; 34)$ representatividade da reprodução no $\mathrm{COE}(\%)=($ reprodução/COE $) \times$ $100 ; 35)$ representatividade da ordenha no $\operatorname{COE}(\%)=($ ordenha/COE $) \times 100 ; 36)$ representatividade dos impostos e taxas no $\mathrm{COE}(\%)=($ impostos e taxas/COE) $\mathrm{x} 100 ; 37)$ representatividade da energia no $\mathrm{COE}(\%)=($ energia/COE $) \times 100 ; 38)$ representatividade das despesas diversas no COE $(\%)=($ despesas diversas/COE) $\mathrm{x}$ 100. Tais índices foram selecionados considerando-se a disponibilidade de dados e a sua relevância. Para a estimação da lucratividade 1 e 2 foi considerada a metodologia proposta por Lopes et $\mathrm{al}^{(9)}$.

Foi realizado o teste de Kolmogorov-Smirnov para se avaliar a distribuição das variáveis contínuas, sendo detectado que algumas das variáveis não apresentaram distribuição normal. Os resultados foram expressos em média \pm desvio padrão (para distribuição normal) e mediana e intervalo de quartis (quando não houve distribuição normal) ${ }^{(10)}$. As comparações foram feitas pelo teste de ANOVA, complementadas pelo teste de Least Significant Difference (LSD) para comparações múltiplas, quando os dados apresentaram distribuição normal. Quando os dados não apresentaram distribuição normal, as comparações entre os grupos foram feitas pelo teste de Kruskal-Wallis, também complementadas pelo teste LSD para comparação múltipla entre as médias ranqueadas das variáveis. Considerou-se a diferença estatística significativa quando $\mathrm{P}<0,05$. O relacionamento entre algumas variáveis foi testado. Todas as análises estatísticas adotadas foram realizadas com o auxílio do software R, versão 2.15.2.

\section{Resultados e discussão}

As quantidades de sistemas de produção foram de $19(31,2 \%), 18(29,5 \%)$ e $24(39,3 \%)$ para mão de obra familiar, mista e contratada, respectivamente. Os indicadores de tamanho e zootécnico dos sistemas de produção de leite em função do tipo de mão de obra utilizada encontram-se descritos na Tabela 1. O valor da mão de obra contratada (MOC) não aparece nulo no grupo mão de obra familiar, pois nele está imputado a quantidade de trabalhadores temporários utilizados durante o período do estudo, como, por exemplo, para a limpeza de pastagens. 


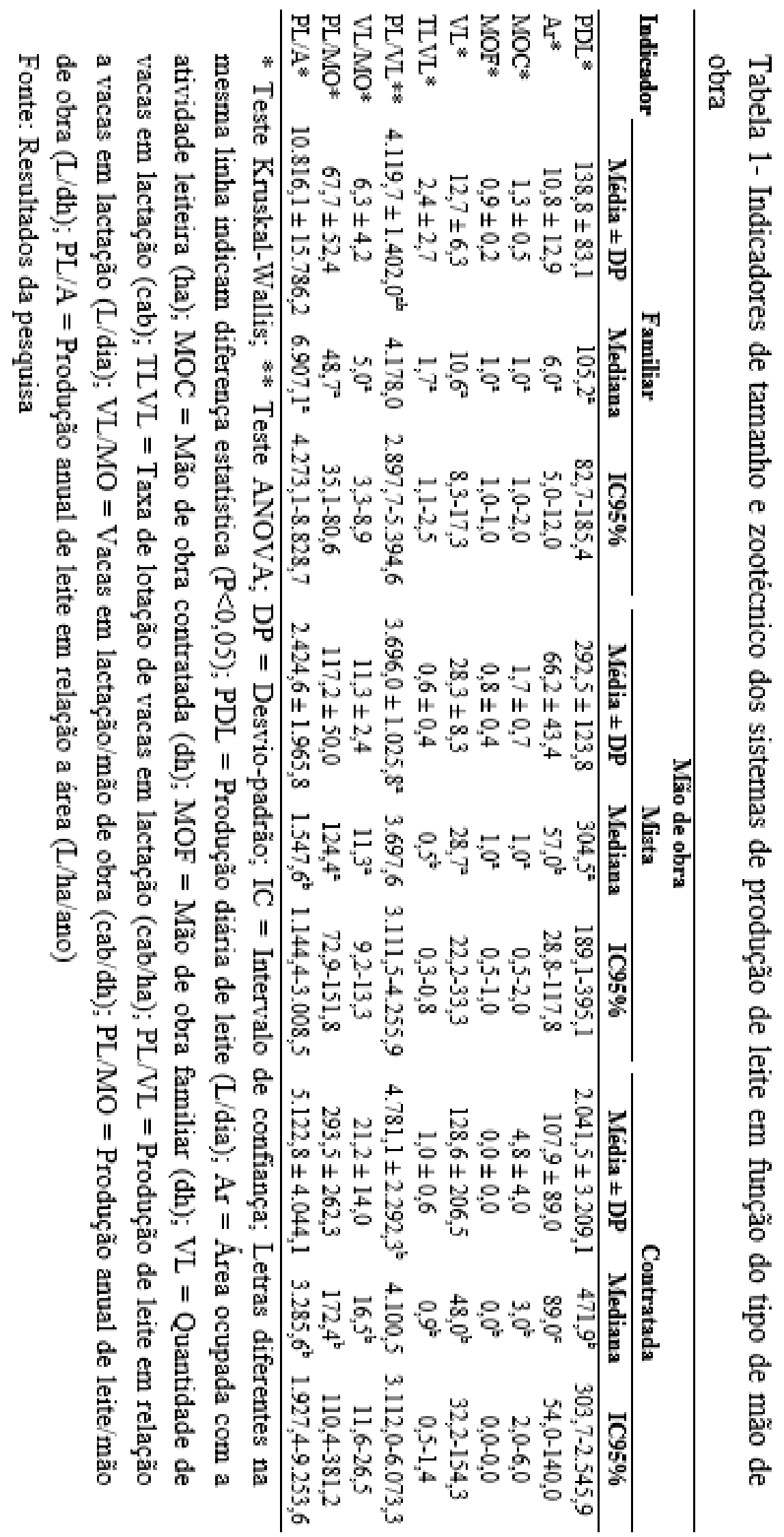


A análise dos indicadores de tamanho e zootécnico dos sistemas de produção caracterizados por mão de obra familiar e mista demonstrou a ocorrência de diferenças estatísticas significativas $(\mathrm{P}<0,05)$ em apenas três dos indicadores (Ar, TLVL e PL/A) (Tabela 1). Isso não era esperado e sugere que a estratificação dos sistemas de produção em função do tipo de mão de obra não foi um bom critério para ser utilizado quando se deseja realizar comparações entre grupos distintos de produtores.

A mediana da produção diária de leite foi de 105,2; 304,5 e 471,9L para mão de obra familiar, mista e contratada, respectivamente. Foi observada diferença estatística significativa $(\mathrm{P}<0,05)$ para mão de obra contratada (Tabela 1). Tal fato pode ser explicado pela maior quantidade de vacas em lactação (48,0 cabeças) e pela maior produtividade por vaca (4.781,1L/vaca/ano), que caracterizaram os produtores que utilizaram mão de obra exclusivamente contratada, participantes desta pesquisa. Por meio da análise de correlação de Pearson, verificou-se que a produção diária de leite correlacionou-se mais com a quantidade de vacas em lactação $(r=0,96)$ e área $(r=0,83)$ que com a produtividade por vaca ao ano $(\mathrm{r}=0,46)$, o que indica que, nos rebanhos estudados, $\mathrm{o}$ volume de produção diário foi mais determinado pelo tamanho da fazenda do que pelos índices de produtividade, sugerindo que fatores ligados à produtividade animal, tais como, padrão genético dos animais e nutrição, poderiam ser priorizados nesses rebanhos.

Os valores da mediana obtidos nesta pesquisa para o indicador área foram de 6,0; 57,0 e 89,0ha para mão de obra familiar, mista e contratada, respectivamente. Houve diferença estatística significativa $(\mathrm{P}<0,05)$ entre os grupos (Tabela 1). A contratação da mão de obra nos rebanhos estudados aumentou com o crescimento da área destinada à atividade leiteira, confirmada pelo coeficiente de correlação de 0,84 entre área e mão de obra contratada, o que indica que essas duas variáveis tendem a aumentar simultaneamente.

A produtividade da mão de obra foi de 48,7; 124,4 e 172,4L/dh para os sistemas de produção com mão de obra familiar, mista e contratada, respectivamente, sendo significativamente maior $(\mathrm{P}<0,05)$ para o grupo mão de obra contratada (Tabela 1). Isso já era esperado, pois, à medida que aumenta a produção diária de leite, a produtividade da mão de obra também é aumentada ${ }^{(11)}$. As menores produtividade e quantidade de vacas em lactação por mão de obra contribuem para a ociosidade da mão de obra, com efeitos não desprezíveis no custo de produção. Esses achados estão de acordo com FAEMG $^{(12)}$ e FAERJ ${ }^{(13)}$, porém diferem de Fassio et al. ${ }^{(14)}$, que observaram produtividade média da mão de obra familiar superior à mão de obra contratada. Nesse sentido, ações poderiam ser tomadas em busca de aumento da produtividade da mão de obra, as quais incluem: capacitação e especialização dos colaboradores, adoção de procedimentos operacionais padrão, adoção de incentivos de desempenho ${ }^{(15)}$, gestão empresarial, com vista à melhoria da eficiência produtiva e alocativa dos fatores de produção, e aumento da escala de produção ${ }^{(16)}$. Além disso, a utilização intensiva de tecnologias pode contribuir favoravelmente para aumentos na produtividade ${ }^{(17,18)}$, uma vez que o baixo uso de capital moderno nas fazendas com mão de obra familiar explica a baixa produtividade e é um dos fatores que limitam o crescimento da produção na maioria dessas fazendas.

No que se refere à produtividade por área, foram observados valores de $10.816,1 ; 2.424,6 \mathrm{e}$ $5.122,8 \mathrm{~L} / \mathrm{ha} /$ ano para mão de obra familiar, mista e contratada, respectivamente. A produção de leite por hectare por ano foi significativamente maior $(\mathrm{P}<0,05)$ para o grupo mão de obra familiar (Tabela 1). Isso pode indicar que, nos sistemas de produção com mão de obra familiar estudados, devido à escassez de terra, observada pelo indicador área, o seu uso foi mais intensivo. Esses 
índices, aliados aos das quantidades medianas de matrizes em lactação por hectare $(1,7 ; 0,5$ e 0,9 , respectivamente) evidenciam que as áreas estão com suas capacidades de utilização ociosas, principalmente nos sistemas de produção com mão de obra mista e contratada. Lopes et al. ${ }^{(19)}$, ao realizarem o levantamento da composição de rebanhos bovinos em sistemas de produção de leite na região de Lavras/MG, constataram que os pecuaristas poderiam otimizar a utilização da terra, em média, 39,75\%. Esses pesquisadores salientaram que, considerando que a remuneração da terra é um importante componente do custo total de produção do leite, uma vez aumentada a taxa de lotação, o fator de produção terra estaria sendo otimizado, reduzindo o custo de produção e aumentando a rentabilidade.

A Tabela 2 mostra os indicadores econômicos dos sistemas de produção distribuídos em função do tipo de mão de obra. Pela análise dos indicadores econômicos, apenas o custo fixo em relação ao custo total e a representatividade da alimentação e da mão de obra no COE e no CT diferiram significativamente $(\mathrm{P}<0,05)$ entre os grupos mão de obra familiar e mista.

A participação da receita bruta do leite na receita bruta total foi de 86,0; 92,6 e 93,4\% para mão de obra familiar, mista e contratada, respectivamente. Não houve diferença estatística significativa ( $P>0,05)$ entre os grupos estudados (Tabela 2). A contribuição de outras receitas, como a venda de animais e de subprodutos de, em média, $13 \%$ pode, segundo Lopes et al. ${ }^{(20)}$, contribuir decisivamente para o melhor desempenho da atividade leiteira. Também é importante salientar que, segundo Lopes et al. ${ }^{(7)}$, para os produtores que não comercializaram subprodutos (esterco), embora em um primeiro momento não signifique acréscimo da receita, representa, também, uma redução nas despesas, quando utilizado na manutenção das capineiras.

A participação do COE na receita bruta total foi de 53,9; 54,0 e 77,2\% para mão de obra familiar, mista e contratada, respectivamente, sendo significativamente maior $(\mathrm{P}<0,05)$ para mão de obra contratada (Tabela 2). Esse fato já era esperado, pois esse grupo de fazendas despendeu mais para o pagamento da mão de obra, item que contribui para o incremento do COE. Somado a isso, fazendas caracterizadas por mão de obra contratada são, em geral, mais tecnificadas e desembolsam quantidade maior de dinheiro para a compra de insumos. Esses resultados evidenciam que muitos esforços gerenciais devem ser feitos com o objetivo de reduzir os custos controláveis, como as despesas referentes à alimentação, mão de obra e sanidade.

O custo fixo é composto pela depreciação, impostos fixos e custo de oportunidade da terra, remuneração do capital investido e do empresário (quando houver). Os valores obtidos para a relação custo fixo e custo total foram de 27,2; 37,1 e 23,6\% para mão de obra familiar, mista e contratada, respectivamente, sendo significativamente maior $(\mathrm{P}<0,05)$ para mão de obra mista (Tabela 2). Tais resultados demonstram a ociosidade da infraestrutura utilizada para exploração leiteira das fazendas caracterizadas por mão de obra mista, evidenciada pelo indicador D/COT, que foi significativamente maior $(\mathrm{P}<0,05)$ para esse grupo de produtores. Uma estratégia que poderia ser buscada por esses produtores baseia-se na expansão do rebanho e/ou aumento da produtividade por vaca e, consequentemente, aumento do volume diário de leite visando a um melhor aproveitamento da infraestrutura disponível e "diluição" dos custos fixos de produção.

A lucratividade consiste em estabelecer um índice percentual para representar o lucro obtido na atividade. Ela é utilizada para comparar atividades iguais, a fim de se conhecer qual é a mais lucrativa. A rentabilidade mede a capacidade da atividade de gerar rendimentos em relação ao capital total disponível, demonstrando uma relação percentual entre o lucro e esse capital. Ela é utilizada para comparar atividades diferentes a fim de verificar qual das atividades possui melhor 
desempenho econômico. Nesta pesquisa, a lucratividade 1 foi de 1,6; -4,5 e 1,8\%, enquanto que, a rentabilidade 1 foi de 0,$5 ;-0,7$ e $0,6 \%$ para mão de obra familiar, mista e contratada, respectivamente. Não houve diferença estatística significativa $(\mathrm{P}>0,05)$ para lucratividade 1 e rentabilidade 1 entre os grupos comparados (Tabela 2).

Tabela 2 Indicadores econômicos dos sistemas de produção de leite em função do tipo de mão de obra

\begin{tabular}{|c|c|c|c|c|c|c|c|c|c|}
\hline \multirow{3}{*}{ Indicador } & \multicolumn{9}{|c|}{ Mão de obra } \\
\hline & \multicolumn{3}{|c|}{ Familiar } & \multicolumn{3}{|c|}{ Mista } & \multicolumn{3}{|c|}{ Contratada } \\
\hline & Média \pm DP & $\begin{array}{c}\text { Medi- } \\
\text { ana }\end{array}$ & IC95\% & Média $\pm \mathbf{D P}$ & $\begin{array}{c}\text { Medi- } \\
\text { ana }\end{array}$ & IC95\% & Média \pm DP & $\begin{array}{c}\text { Medi- } \\
\text { ana }\end{array}$ & IC95\% \\
\hline$\overline{\mathrm{RBL} / \mathrm{RBT}^{*}}$ & $85,5 \pm 8,3$ & 86,0 & $80,9-92,4$ & $89,5 \pm 10,9$ & 92,6 & $84,5-98,3$ & $86,9 \pm 14,4$ & 93,4 & $79,0-98,5$ \\
\hline $\mathrm{COE} / \mathrm{RBT}^{*}$ & $56,0 \pm 15,0$ & $53,9^{\mathrm{a}}$ & $44,9-62,0$ & $58,1 \pm 13,1$ & $54,0^{\mathrm{a}}$ & $48,4-66,0$ & $76,0 \pm 20,2$ & $77,2^{\mathrm{b}}$ & $59,2-90,0$ \\
\hline COT/RBT* & $80,1 \pm 17,1$ & 77,9 & $68,4-84,5$ & $74,5 \pm 14,5$ & 67,1 & $62,2-86,4$ & $83,4 \pm 21,9$ & 82,9 & $65,5-101,7$ \\
\hline COEun/PL** & $65,7 \pm 16,5^{\mathrm{a}}$ & 62,0 & $53,0-71,7$ & $65,7 \pm 15,5^{\mathrm{a}}$ & 63,3 & $50,8-75,4$ & $41,0 \pm 12,0^{b}$ & 84,2 & $77,0-97,5$ \\
\hline COTun/PL* & $93,9 \pm 18,8$ & 90,6 & $77,6-103,6$ & $84,4 \pm 18,2$ & 80,4 & $67,7-103,3$ & $96,8 \pm 23,4$ & 90,8 & $84,0-106,5$ \\
\hline CTun/PL* & $117,5 \pm 27,1$ & 110,7 & $94,5-126,9$ & $118,5 \pm 24,3$ & 114,9 & $96,1-143,4$ & $121,2 \pm 41,4$ & 112,7 & $98,0-127,6$ \\
\hline $\mathrm{CF} / \mathrm{CT}^{* * *}$ & $27,2 \pm 8,8^{\mathrm{a}}$ & 25,2 & $23,3-31,5$ & $37,1 \pm 12,0^{b}$ & 37,6 & $30,0-46,0$ & $23,6 \pm 13,2^{a}$ & 23,4 & $11,5-32,9$ \\
\hline $\mathrm{D} / \mathrm{COT}^{* *}$ & $11,9 \pm 3,7^{\mathrm{ab}}$ & 11,9 & $10,6-13,3$ & $14,5 \pm 7,4^{\mathrm{a}}$ & 14,3 & $8,7-18,4$ & $8,9 \pm 5,6^{b}$ & 7,7 & $4,1-11,8$ \\
\hline L $1^{*}$ & $-0,2 \pm 23,9$ & 1,6 & $-5,7-18,3$ & $-5,0 \pm 20,4$ & $-4,5$ & $-17,2-14,7$ & $-3,7 \pm 33,5$ & 1,8 & $-14,8-20,8$ \\
\hline $\mathrm{L} 2 *$ & $19,9 \pm 17,1$ & 22,1 & $15,5-31,6$ & $25,5 \pm 14,5$ & 32,9 & $13,6-37,8$ & $16,6 \pm 21,9$ & 17,1 & $-1,7-34,5$ \\
\hline $\mathrm{R} 1^{*}$ & $1,6 \pm 9,1$ & 0,5 & $-3,6-8,3$ & $-0,4 \pm 6,5$ & $-0,7$ & $-5,6-6,3$ & $2,6 \pm 10,3$ & 0,6 & $-4,9-10,3$ \\
\hline $\mathrm{R} 2 * *$ & $8,0 \pm 9,0$ & 7,1 & $3,0-14,4$ & $10,3 \pm 7,9$ & 9,3 & $3,3-15,8$ & $9,5 \pm 11,4$ & 6,4 & $-0,5-17,0$ \\
\hline $\mathrm{A} / \mathrm{CT}^{* *}$ & $38,5 \pm 8,5^{\mathrm{a}}$ & 40,7 & $32,3-44,0$ & $31,6 \pm 8,8^{b}$ & 30,8 & $5,6-14,4$ & $41,0 \pm 12,0^{\mathrm{a}}$ & 41,1 & $10,6-19,3$ \\
\hline $\mathrm{MO} / \mathrm{CT}^{* *}$ & $3,1 \pm 3,9^{\mathrm{a}}$ & 0,8 & $0,0-17,8$ & $10,1 \pm 5,2^{\mathrm{b}}$ & 8,5 & $18,8-57,0$ & $14,5 \pm 5,4^{c}$ & 14,6 & $25,5-59,7$ \\
\hline $\mathrm{S} / \mathrm{CT} * *$ & $3,4 \pm 1,9^{\mathrm{ab}}$ & 3,1 & $1,9-4,9$ & $2,7 \pm 1,3^{\mathrm{a}}$ & 2,4 & $1,9-3,3$ & $4,2 \pm 2,7^{b}$ & 3,8 & $2,3-4,9$ \\
\hline $\mathrm{R} / \mathrm{CT}^{*}$ & $0,4 \pm 0,9$ & $0,0^{\mathrm{a}}$ & $0,0-0,2$ & $0,1 \pm 0,2$ & $0,0^{\mathrm{a}}$ & $0,0-0,0$ & $1,2 \pm 1,5$ & $0,6^{\mathrm{b}}$ & $0,3-1,6$ \\
\hline $\mathrm{O} / \mathrm{CT}^{*}$ & $0,5 \pm 0,6$ & $0,4^{\mathrm{a}}$ & $0,0-1,0$ & $0,3 \pm 0,3$ & $0,3^{\mathrm{a}}$ & $0,1-0,6$ & $1,3 \pm 1,2$ & $1,1^{b}$ & $0,1-2,3$ \\
\hline $\mathrm{IT} / \mathrm{CT}^{*}$ & $0,2 \pm 0,4$ & 0,0 & $0,0-0,2$ & $0,4 \pm 0,4$ & 0,4 & $0,0-0,6$ & $0,7 \pm 1,6$ & 0,2 & $0,1-0,5$ \\
\hline $\mathrm{E} / \mathrm{CT}^{* *}$ & $3,7 \pm 2,1$ & 3,8 & $1,8-4,8$ & $4,7 \pm 2,5$ & 4,5 & $2,4-7,2$ & $3,8 \pm 2,2$ & 3,3 & $2,4-4,7$ \\
\hline $\mathrm{DD} / \mathrm{CT}^{*}$ & $7,1 \pm 5,1$ & 7,7 & $2,3-11,0$ & $6,5 \pm 4,5$ & 4,8 & $4,0-8,2$ & $8,3 \pm 4,1$ & 8,5 & $4,9-11,4$ \\
\hline $\mathrm{A} / \mathrm{COE}^{* *}$ & $67,9 \pm 8,2^{\mathrm{a}}$ & 65,4 & $62,5-76,1$ & $55,9 \pm 8,9^{b}$ & 57,2 & $46,4-60,9$ & $53,7 \pm 09,9^{b}$ & 55,8 & $47,1-59,1$ \\
\hline $\mathrm{MO} / \mathrm{COE}^{* *}$ & $5,1 \pm 6,2^{\mathrm{a}}$ & 1,7 & $0,0-11,0$ & $18,1 \pm 9,2^{b}$ & 15,2 & $11,4-26,5$ & $20,5 \pm 10,0^{b}$ & 17,7 & $14,2-25,6$ \\
\hline $\mathrm{S} / \mathrm{COE}^{*}$ & $5,8 \pm 2,8$ & 5,6 & $3,5-8,9$ & $4,7 \pm 1,9$ & 4,7 & $3,9-5,6$ & $5,4 \pm 2,9$ & 5,1 & $3,0-6,6$ \\
\hline $\mathrm{R} / \mathrm{COE}^{*}$ & $0,6 \pm 1,5$ & $0,0^{\mathrm{a}}$ & $0,0-0,3$ & $0,1 \pm 0,3$ & $0,0^{\mathrm{a}}$ & $0,0-0,1$ & $1,4 \pm 1,6$ & $0,9^{b}$ & $-0,4-2,1$ \\
\hline $\mathrm{O} / \mathrm{COE}^{*}$ & $0,9 \pm 0,9$ & $0,0^{\mathrm{a}}$ & $0,6-1,5$ & $0,6 \pm 0,5$ & $0,2^{\mathrm{a}}$ & $0,4-1,0$ & $1,6 \pm 1,4$ & $0,1^{\mathrm{b}}$ & $1,5-2,8$ \\
\hline $\mathrm{IT} / \mathrm{COE}^{*}$ & $0,3 \pm 0,6$ & 0,0 & $0,0-0,3$ & $0,8 \pm 0,9$ & 0,0 & $0,6-1,3$ & $0,8 \pm 1,9$ & 0,3 & $0,1-0,7$ \\
\hline $\mathrm{E} / \mathrm{COE}^{*}$ & $6,7 \pm 3,9$ & $6,4^{\mathrm{ab}}$ & $2,9-8,5$ & $8,8 \pm 5,2$ & $9,0^{\mathrm{a}}$ & $4,2-12,2$ & $5,3 \pm 3,3$ & $4,2^{b}$ & $3,0-6,9$ \\
\hline $\mathrm{DD} / \mathrm{COE}^{* *}$ & $12,6 \pm 08,7$ & 13,3 & $4,6-18,6$ & $11,0 \pm 5,4$ & 8,8 & $6,8-14,6$ & $11,2 \pm 4,9$ & 11,7 & $6,2-15,0$ \\
\hline
\end{tabular}

* Teste Kruskal-Wallis; ** Teste ANOVA; DP = Desvio-padrão; IC = Intervalo de confiança; Letras diferentes na mesma linha indicam diferença estatística $(\mathrm{P}<0,05) ; \mathrm{RBL} / \mathrm{RBT}=$ Receita bruta do leite em relação à receita bruta total $(\%) ; \mathrm{COE} / \mathrm{RBT}=\mathrm{COE} /$ receita bruta total $(\%) ; \mathrm{COT} / \mathrm{RBT}=\mathrm{COT} /$ receita bruta total $(\%) ; \mathrm{COEun} / \mathrm{PL}=\mathrm{COE}$ unitário/preço de venda do leite (\%); COTun/PL = COT unitário/preço de venda do leite (\%); CTun/PL = Custo total unitário/preço de venda do leite (\%); $\mathrm{CF} / \mathrm{CT}=$ Relação entre o custo fixo e o custo total (\%); $\mathrm{D} / \mathrm{COT}=$ Depreciação/COT (\%); L 1 = Lucratividade 1 (\%); L 2 = Lucratividade 2 (\%); R 1 = Rentabilidade 1 (\%); R 2 = Rentabilidade 2 (\%); $\mathrm{A} / \mathrm{CT}=$ Representatividade da alimentação no custo total (\%); $\mathrm{MO} / \mathrm{CT}=$ Representatividade da mão de obra no custo total (\%); $\mathrm{S} / \mathrm{CT}=$ Representatividade da sanidade no custo total (\%); $\mathrm{R} / \mathrm{CT}=$ Representatividade da reprodução no custo total (\%); $\mathrm{O} / \mathrm{CT}=$ Representatividade da ordenha no custo total (\%); IT/CT = Representatividade dos impostos e taxas no custo total (\%); E/CT = Representatividade da energia no custo total (\%); $\mathrm{DD} / \mathrm{CT}=$ Representatividade das despesas diversas no custo total (\%); $\mathrm{A} / \mathrm{COE}=$ Representatividade da alimentação no $\mathrm{COE}$ (\%); $\mathrm{MO} / \mathrm{COE}=$ Representatividade da mão de obra no $\mathrm{COE}$ (\%); $\mathrm{S} / \mathrm{COE}=$ Representatividade da sanidade no $\mathrm{COE}(\%) ; \mathrm{R} / \mathrm{COE}=$ Representatividade da reprodução no $\mathrm{COE}$ (\%); $\mathrm{O} / \mathrm{COE}=$ Representatividade da ordenha no $\mathrm{COE}$ (\%); IT/COE = Representatividade dos impostos e taxas no $\mathrm{COE}(\%) ; \mathrm{E} / \mathrm{COE}=$ Representatividade da energia no $\mathrm{COE}$ (\%); $\mathrm{DD} / \mathrm{COE}=$ Representatividade das despesas diversas no $\mathrm{COE}$ (\%); Fonte: Resultados da pesquisa 
Para o cálculo da lucratividade 1 e rentabilidade 1, levou-se em consideração o resultado da atividade, já estando imputada a remuneração do capital e da terra. Visando maior clareza na análise dos resultados, foi considerada ainda a lucratividade 2 e a rentabilidade 2, que consistem no uso da margem líquida, ao invés do lucro líquido ${ }^{(9)}$. Ao considerar a margem líquida (Receita - Custo operacional total), o valor obtido poderá ser comparado com a caderneta de poupança, por exemplo, ou qualquer outra aplicação, conferindo maior poder de comparação. No presente estudo, a lucratividade 2 foi de 22,1; 32,9 e 17,1\%, enquanto que a rentabilidade 2 representou 8,0; 10,3 e 9,5\% para mão de obra familiar, mista e contratada, respectivamente, não havendo diferença estatística significante $(\mathrm{P}>0,05)$ para esses indicadores entre os grupos comparados (Tabela 2). Isso denota que o resultado econômico obtido foi independente do tipo de mão de obra utilizada pelo sistema de produção. Os maiores índices de produtividade e o maior capital imputado no sistema de produção, incluindo mão de obra, em muitos casos simplesmente implica em maior produção leiteira e não necessariamente maior eficiência produtiva ${ }^{(17)}$. Tal achado confirma que vários fatores podem determinar o desempenho econômico em rebanhos leiteiros, o que, segundo Juszczyk ${ }^{(21)}$, é compreensível devido à natureza complexa e multifatorial da atividade leiteira.

Cabe ressaltar ainda que, embora os resultados econômicos não tenham sido significativamente diferentes entre os grupos de produtores, há o problema da ineficiência de escala de produção. Isso sinaliza oportunidade de crescimento das pequenas propriedades, caracterizadas por mão de obra familiar e mista, por meio da busca de maior eficiência produtiva, com variação proporcionalmente menor do custo de produção em relação à produção de leite, indicando taxas de rendimento crescentes à escala de produção ${ }^{(16,22,23)}$.

Nascimento et al. ${ }^{(24)}$, ao analisarem produtores de leite no estado de Minas Gerais, observaram que o percentual de mão de obra familiar utilizado na produção é fator determinante para explicar as diferenças entre os níveis de eficiência técnica, sendo que aqueles produtores que empregam maior percentual de mão de obra familiar são também aqueles de menor eficiência técnica. Cabrera et al. ${ }^{(25)}$, trabalhando com 273 rebanhos do Wisconsin, EUA, concluíram que entre as variáveis que mais se correlacionaram com a lucratividade está a participação da mão de obra familiar nas tarefas diárias da fazenda, o que, segundo $\operatorname{Carter}^{(26)}$, deve-se ao fato de que, na produção agrícola, os membros da família procuram maximizar o bem-estar da família, em vez do bem-estar individual e, consequentemente, proporcionar um maior esforço para a produção. Essa conclusão difere daquela encontrada no trabalho de Hallan \& Machado $^{(27)}$ sobre a atividade leiteira portuguesa, em que a variável mão de obra familiar não foi importante para explicar a eficiência técnica dos produtores. A diferença entre os resultados encontrados na literatura ressalta a importância de se considerarem os fatores que sejam comuns aos sistemas de produção e que podem estar associados à lucratividade, uma vez que estimativas baseadas apenas na média amostral pode representar um universo muito heterogêneo e não traduzir a realidade das fazendas leiteiras. No entanto, a estratificação dos sistemas de produção em função do tipo de mão de obra parece não ser um bom critério para ser utilizado quando se deseja realizar comparações entre grupos distintos de produtores. Este achado, apresentado no presente estudo, sugere pesquisas que busquem a caracterização de indicadores estratificados de desempenho e sua correlação com a eficiência econômica em outras regiões do país e a investigação de outros critérios para a análise entre grupos distintos de produtores de leite, tal como o fator escala de produção, de obtenção mais simples e, provavelmente, de maior acurácia.

A divisão das despesas em grupos possibilita o monitoramento e uma análise mais detalhada, 
auxiliando o produtor e o técnico a encontrarem possíveis pontos de estrangulamento ${ }^{(19)}$. A representatividade dos itens componentes do custo total e operacional efetivo foram, em ordem decrescente, alimentação e mão de obra, com exceção do grupo de produtores caracterizados por mão de obra familiar, que foi alimentação e despesas diversas. Os valores obtidos para representatividade da alimentação no COE foram de 67,9; 55,9 e 53,7\% para mão de obra familiar, mista e contratada, respectivamente. $O$ grupo de despesas alimentação foi significativamente maior $(\mathrm{P}<0,05)$ para mão de obra familiar. Isso pode ser explicado, possivelmente, pelo uso mais racional dos insumos utilizados para alimentação animal pelos sistemas de produção que utilizavam mão de obra mista e contratada e pelo fato da representatividade do item alimentação no COE crescer com a diminuição da contratação de mão de obra por efeito da propriedade de grandezas proporcionais, em que há uma contribuição menor de outros itens, como mão de obra e sanidade, nos grupos de sistemas de produção com mão de obra familiar. Em relação ao CT, a representatividade da alimentação foi de 38,5; 31,6 e 41,0\% para mão de obra familiar, mista e contratada, respectivamente, sendo significativamente maior para mão de obra familiar e contratada (Tabela 2). A diminuição da representatividade da alimentação para o grupo mão de obra familiar, quando considerado o CT, em comparação ao COE, deveu-se a contribuição da remuneração da mão de obra familiar.

Os gastos referentes à mão de obra representam o dispêndio com a remuneração da mão de obra permanente e a contratação de trabalhadores temporários para a realização de atividades esporádicas. Esses gastos representou 5,1; 18,1 e 20,5\% do COE para mão de obra familiar, mista e contratada, respectivamente, sendo significativamente menor $(\mathrm{P}<0,05)$ para o grupo mão de obra familiar (Tabela 2), o que já era esperado. Apesar da pequena contribuição verificada para o grupo mão de obra familiar, é importante considerá-la em estudos de análise econômica de produção de leite pois, no Brasil, a proporção da mão de obra familiar decresce à medida que a escala de produção aumenta $^{(12,14)}$; entretanto, em alguns países, como os Estados Unidos, por exemplo, essa proporção é alta, mesmo nas grandes fazendas ${ }^{(28)}$.

$\mathrm{O}$ resultado da atividade leiteira depende da forma como são gerenciados e alocados os recursos de produção $^{(16)}$. O item mão de obra tem grande participação nos custos da atividade leiteira. A redução dos gastos com o fator trabalho pode colaborar para melhorar o resultado econômico do sistema. No Brasil, a estratégia comumente utilizada para reduzir os custos com a mão de obra se constitui na prática de baixos salários. Por outro lado, a otimização e melhor eficiência técnica da mão de obra poderia permitir a redução dos custos e o pagamento de salários mais justos aos funcionários.

Despesas com sanidade, reprodução e ordenha foram significativamente maiores $(\mathrm{P}<0,05)$ para $\mathrm{O}$ grupo mão de obra contratada (Tabela 2), possivelmente, devido ao maior controle sanitário do rebanho, uso de inseminação artificial e ordenhadeira mecânica por esse grupo de produtores. Não obstante, tiveram baixa representatividade no $\mathrm{COE}$, o que evidencia que não se justifica concentrar grandes esforços gerenciais e tecnológicos objetivando reduzir tais valores, nem mesmo deixar de utilizar alguns insumos considerados importantes, tais como aqueles destinados a saúde animal, cujos impactos na produtividade seriam altos e, no custo de produção, insignificantes. 


\section{Conclusões}

Índices de medição de desempenho de referências devem ser considerados levando-se em conta os fatores que sejam comuns aos sistemas de produção, com objetivo de gerar parâmetros mais precisos para a atividade leiteira, considerando a grande heterogeneidade da mesma no território brasileiro. No entanto, a estratificação dos sistemas de produção em função do tipo de mão de obra pode não ser um bom critério para ser utilizado quando se deseja realizar comparações entre grupos distintos de produtores.

A estimativa de índices de desempenho zootécnico e econômico, como instrumento de rotina, é uma ferramenta gerencial imprescindível para se verificar a viabilidade operacional e econômica do sistema de produção e fornecer com acurácia as informações necessárias para o planejamento e a tomada de decisão. O tipo de mão de obra utilizada não parece determinar a eficiência econômica do sistema de produção. Os itens componentes do custo total e operacional efetivo que exerceram maiores representatividades foram, em ordem decrescente, alimentação e mão de obra, para os grupos mão de obra contratada e mista, e alimentação e despesas diversas, para o grupo mão de obra familiar.

\section{Agradecimentos}

Os autores agradecem à Fundação de Amparo à Pesquisa do Estado de Minas Gerais (FAPEMIG) pelo apoio recebido para a realização desta pesquisa (SHA APQ 01974/11).

\section{Referências}

1. Martins MC. Competitividade da cadeia produtiva do leite no Brasil. Revista de Política Agrícola. 2004 Jul; 3: 38-71.

2. IBGE - Instituto Brasieliro de Geografia e Estatística. 2010. Disponível em: http://www.sidra.ibge.gov.br/bda/tabela/protabl.asp?c=74\&z=t\&o=23\&i=P. Acesso em: 28 mar 2012.

3. Fundação Getúlio Vargas. Rio de Janeiro: 2012. Disponível em: http://www.antigofgvdados.fgv.br/dsp_frs_pai_ferramentas.asp. Acesso em: 15 set 2012.

4. Alves E, Souza GS, Brandão ASP. A situação do produtor com menos de cem hectares. Revista de Política Agrícola, 2001 Jan; 1: 27-36.

5. Gonçalves RML, Vieira WC, Lima JE, Gomes ST. Analysis of technical efficiency of milk-producing farms in Minas Gerais. Economia Aplicada. 2008 Apr; 12 (2): 321-35.

6. Oliveira AS, Cunha DNFV, Campos JMS, Vale SMLR, Assis AJ. Identificação e quantificação de indicadores-referência de sistemas de produção de leite. Revista Brasileira de Zootecnia. 2007; 36 (2): $507-$ 16.

7. Lopes MA, Dias AS, Carvalho FM, Lima ALR, Cardoso MG, Carmo EA. Efeito do tipo de mão-de-obra nos resultados econômicos de sistemas de produção de leite na região de Lavras (MG) nos anos 2004 e 2005. Revista Brasileira de Agrociencia. 2010 Jan; 16: 125-32.

8. Lopes MA, Lima ALR, Carvalho FM, Reis RP, Santos IC, Saraiva FH. Controle gerencial e estudo da rentabilidade de sistemas de produção de leite na região de Lavras (MG). Ciência e Agrotecnologia. 2004 Jul; 28 (4): 883-92.

9. Lopes MA, Santos G, Resende MC, Carvalho FM, Cardoso MG. Estudo da rentabilidade de sistemas de 
produção de leite na região de Nazareno (MG). Ciência Animal Brasileira. 2011 Jan; 12 (1): 58-69.

10. Duquia RP, Bastos JLD. Medidas de tendência central: onde a maior parte dos indivíduos se encontra? Scientia Medica. 2006; 16 (4): 190-94.

11. Bewley J, Palmer RW, Jackson-Smith DB. Modeling milk production and labor efficiency in modernized Wisconsin dairy herds. Journal of Dairy Science. 2001 Mar; 84 (3): 705-16.

12. FEDERAÇÃO DE AGRICULTURA DO ESTADO DE MINAS GERAIS. Diagnóstico da pecuária leiteira do estado de Minas Gerais em 2005: relatório de pesquisa. Belo Horizonte: 2006. 156 p. Disponível em http://cigeneticabovina.com.br/downloads/1647f84f-diagnostico\%20pecuaria\%20mineira.pdf, zcesso em abril de 2015.

13. FEDERAÇÃO DE AGRICULTURA DO ESTADO DO RIO DE JANEIRO. Diagnóstico da cadeia produtiva do leite do estado do Rio de Janeiro. Rio de Janeiro: 2010. 180 p. Disponível em http://sistemafaerj.com.br/baldecheio/wp-content/uploads/2014/05/diagnostico-cadeia-produtiva-leite-

2010.pdf, acesso em abril de 2015.

14. Fassio LH, Reis RP, Geraldo LG. Desempenho técnico e econômico da atividade leiteira em Minas Gerais. Ciência e Agrotecnologia. 2006 Nov; 30 (6): 1154-61.

15. Stup RE, Hyde J, Holden LA. Relationships between selected human resource management practices and dairy farm performance. Journal of Dairy Science. 2006 Mar; 89 (3): 1116-20.

16. Marques VM, Reis RP, Sáfadi T, Reis AJ. Custos e escala na pecuária leiteira: estudo de casos em Minas Gerais. Ciência e Agrotecnologia. 2002 Sep; 26 (5): 1027-34.

17. Kompas T, Che TN. Technology choice and efficiency on Australian dairy farms. Australian Journal of Agricultural and Resource Economics. 2006; 50: 65-83.

18. Alvarez A, Corral J, Solís D, Pérez JA. Does intensification improve the economic efficiency of dairy farms? Journal of Dairy Science. 2008 Sep; 91 (9): 3693-8.

19. Lopes MA, Cardoso MG, Dias AS, Carmo EAD. Efeito da escala de produção nos resultados econômicos de sistema de produção de leite na região de Lavras (MG) em 2004 e 2005. Archivos Latinoamericanos de Producción Animal. 2008; 16 (3): 121-9.

20. Lopes MA, Santos G, Carvalho FM. Comparativo de indicadores econômicos da atividade leiteira de sistemas intensivos de produção de leite no Estado de Minas Gerais. Revista Ceres. 2012 Jul; 59(4): 458-65.

21. Juszczyk S. Milk production profitability: multiple regression analysis. Electronic Journal of Polish Agricultural Universities [Internet]. 2005; 8 (4): [about 6 p.]. Disponível em: http://www.ejpau.media.pl/volume8/issue4/art- 46.html. Acesso em: 25 nov 2013.

22. Lopes PF, Reis RP, Yamaguchi LCT. Custos e escala de produção na pecuária leiteira: estudo nos principais estados produtores do Brasil. Revista de Economia e Sociologia Rural. 2007 Jul; 45 (3): 567-90.

23. Mosheim R, Lovell CAK. Scale economies and inefficiency of U.S. dairy farms. American Journal of Agricultural Economics. 2009 Aug; 91 (3): 777-94.

24. Nascimento ACC, Lima JE, Braga MJ, Nascimento M, Gomes AP. Eficiência técnica da atividade leiteira em Minas Gerais: uma aplicação de regressão quantílica. Revista Brasileira de Zootecnia. 2012; 41 (3): 783-89.

25. Cabrera VE, Solís D, Corral J. Determinants of technical efficiency among dairy farms in Wisconsin. Journal of Dairy Science. 2010 Jan; 93 (1): 387-93.

26. Carter MR. Resource allocation and use under collective rights and labour management in Peruvian coastal agriculture. Economic Journal. 1984 Dec; 94 (376): 826-46.

27. Hallam D, Machado F. Efficiency analysis with panel data: a study of Portuguese dairy farms. European Review of Agricultural Economics. 1996; 23 (1): 79-93.

28. Tauer LW, Belbase KP. Technical efficiency of New York dairy farms. Northeast Journal of Agricultural Resource Economics. 1987; 16 (1): 10-6. 